\title{
Coronavirus: Was bisher über SARS-CoV-2 bekannt ist
}

\author{
Marion Anna Becker
}

Seit dem ersten Auftreten der Coronavirus-Krankheit 2019 (COVID-19) in China sind nur wenige Wochen vergangen. In dieser Zeit hat sich die Infektion weltweit ausgebreitet. Doch auch das Wissen über das neuartige Coronavirus (SARS-CoV-2) ist gewachsen. Dieser Artikel fasst die wichtigsten Erkenntnisse zusammen und gibt einen Überblick, wie die limitierte Datenlage hinsichtlich Schwangerschaft und Geburt einzuschätzen ist. (Stand: 21.03.2020)

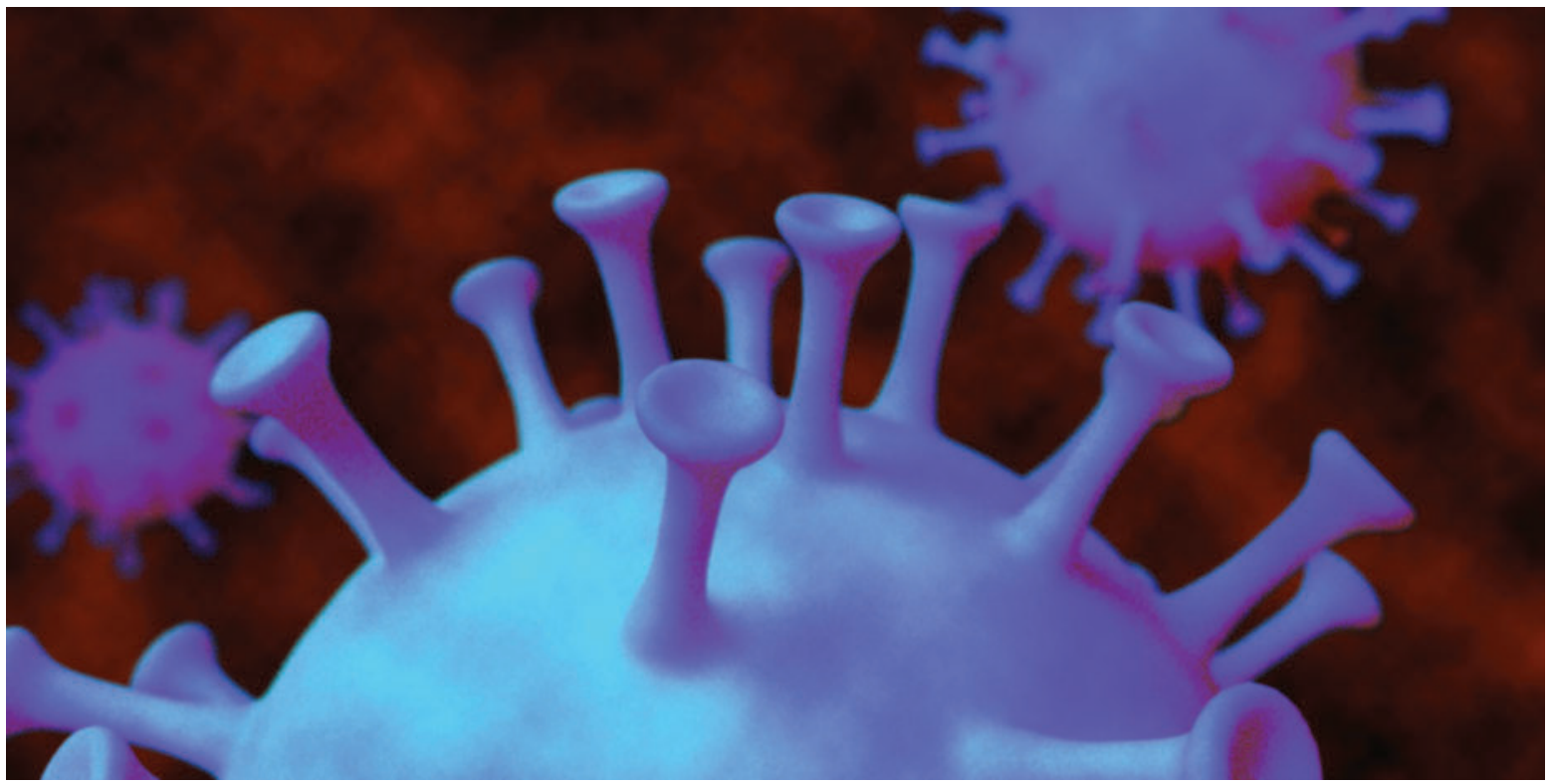

Abb. 1 Schematische Darstellung von SARS-CoV-2: Die Proteinstrukturen auf der Virushülle geben Coronaviren ihr typisches kranzförmiges Aussehen. (Illustration: Thieme Gruppe)

\section{Entstehung und Verbreitung}

Ende Dezember 2019 meldeten Gesundheitsbehörden in China mehrere Fälle von Pneumonien unbekannter Ätiologie. Chinesische Wissenschaftler führten diese auf ein neuartiges Coronavirus zurück, das sich durch große Homologie zum Erreger von SARS (Severe Acute Respiratory Syndrome) auszeichnet und daher als SARS-Coronavirus-2 (SARS-CoV-2) bezeichnet wurde [6][9][14].

\section{INFO}

\section{Coronaviren}

Coronaviren sind RNA-Viren, die sowohl Tiere als auch Menschen befallen können. Ihr Name ist auf ihr typisches kranzförmiges Aussehen (lat. Corona; Kranz, Krone) zurückzuführen. Beim Menschen lösen die Viren vor allem Erkrankungen des Respirationstrakts aus. Neben SARS-CoV und dem neuartigen Coronavirus SARS-CoV-2 gehört auch der Erreger des Middle East Respiratory Syndrome (MERS) zur Familie der Coronaviren. Alle drei können neben milden und moderaten Symptomen auch sehr schwere Krankheitsverläufe nehmen. Vier weitere Coronaviren sind in Deutschland verbreitet. Sie führen zu typischen Erkältungssymptomen, die in der Regel mild verlaufen [9][5]. 
Das erste Auftreten von COVID-19 wird mit der chinesischen Stadt Wuhan in der Provinz Hubei in Zusammenhang gebracht, da dort bis Mitte März 2020 die meisten Infektionen mit COVID-19 gemeldet wurden. Seither ist die Zahl der Infizierten außerhalb Chinas stark angestiegen, während die gemeldeten Neuinfektionen aus China zurückgehen [6][9]. Am 11. März 2020 stufte die Weltgesundheitsorganisation (WHO) die weltweite Ausbreitung von COVID-19 als Pandemie ein [16].

\section{Prävention und Therapie}

Die Erkrankung gilt als sehr infektiös, da sich das Virus in den oberen Atemwegen repliziert und von dort leicht von Mensch zu Mensch übertragen werden kann. In 4 von 5 Fällen ist der Verlauf von COVID-19 mild, doch insbesondere Risikogruppen können von schweren Krankheitsverläufen betroffen sein und an den Folgen der Infektion versterben. Bei vielen der schwer erkrankten Patienten muss mit einer im Vergleich zu anderen akuten respiratorischen Infektionen längeren intensivmedizinischen Behandlung und Beatmungszeit bzw. mit zusätzlichem Sauerstoffbedarf gerechnet werden. Dadurch kann das Gesundheitssystem überlastet werden. Da zum jetzigen Zeitpunkt weder eine Impfung noch eine spezifische Therapie zur Verfügung stehen, gilt als wichtigste Maßnahme, die Verbreitung der Krankheit zu verlangsamen. Dabei wirken drei zentrale Komponenten zusammen:

- Verhinderung der Ausbreitung durch Fallfindung und Absonderung von engen Kontaktpersonen

- Soziale Distanz schaffen

- Gezielter Schutz von vulnerablen Gruppen [10]

\section{WEB-TIPP}

Freie Beatmungsplätze für Patienten bereitstellen und finden

Seit Mitte März 2020 ist das DIVI Intensivregister (DIVI: Deutsche Intensivmedizinische Vereinigung für Intensiv- und Notfallmedizin) online. Krankenhäuser, deren Beatmungsplätze ausgelastet sind, sollen dort schnell eine Klinik finden, an die sie COVID-19-Patienten weiterleiten können (13). Mehr Infos unter www.divi.de/register/intensivregister

\section{Übertragungswege}

Als Hauptübertragungsweg ist nach jetzigem Kenntnisstand von einer Tröpfcheninfektion auszugehen. Eine Übertragung durch Schmierinfektion und kontaminierte Oberflächen und über Konjunktiven ist ebenfalls nicht auszuschließen. Auch PCR-positive Stuhlproben wurden bei Patienten mit COVID-19 gefunden. Bisher gibt es jedoch keine gesicherten Beweise für vermehrungsfähige Viren und damit für eine Ansteckungsgefahr über den Stuhl [14].
In einer Publikation des Royal College of Obstetricians and Gynaecologists (RCOG) vom 21. März 2020 weisen die Verfasser darauf hin, dass Beobachtungsstudien aus China keine Beweise für eine vertikale Übertragung des Virus vor oder während der Geburt erbracht hätten [15]. In einer Fallserie, die von Chen et al. veröffentlicht wurde, wurden Fruchtwasser, Nabelschnurblut, Rachenabstriche des Neugeborenen und Muttermilchproben der infizierten Mütter negativ auf SARS-CoV-2 getestet [2]. In einer weiteren Fallserie wurden Abstriche der Plazenta von drei Müttern mit COVID-19 entnommen und negativ auf das Virus getestet [3]. Diese ersten Ergebnisse legen eine postnatale Übertragung des Virus nahe. Aufgrund der stark limitierten Datenlage sind sie jedoch vorsichtig zu bewerten [15].

\section{INFO}

COVID-19

Die Erkrankung verläuft sehr unterschiedlich und äußert sich häufig mit unspezifischen Symptomen, die einer Erkältung oder Grippe sehr ähnlich sein können [14].
Häufige Symptome:
- Fieber
- Husten
Weitere Symptome:
- Kurzatmigkeit
- Muskel-/Gelenkschmerzen
- Halsschmerzen
- Kopfschmerzen
- Übelkeit/Erbrechen
- Verstopfte Nase
- Durchfall

Inkubationszeit:

Von der Ansteckung bis zum ersten Auftreten der Symptome vergehen 1-14 Tage (Median: 5-6 Tage). Krankheitsverlauf:

Die Datenlage zur Symptomatik und zum Krankheitsverlauf der in Deutschland aufgetretenen Fälle ist noch begrenzt [14]. Bei 55924 laborbestätigten Fällen aus China verliefen $80 \%$ der Erkrankungen mild bis moderat, $14 \%$ verliefen schwer, aber nicht lebensbedrohlich (mit Atemnot, Sauerstoffsättigung <94\% oder Lungeninfiltraten in mehr als der Hälfte der Lunge), 6\% waren kritische bis lebensbedrohliche Fälle (mit Lungenversagen, septischem Schock oder multiplem Organversagen) [14][17].

\section{Risikogruppen für schwere Verläufe:}

- Ältere Personen ab 50-60 Jahren

- Raucher

- Patienten mit Vorerkrankungen der Lunge, des Herzens, mit chronischen Lebererkrankungen, mit Diabetes mellitus, mit Krebserkrankungen, mit geschwächtem Immunsystem [14] 


\section{Auswirkungen auf die Schwangerschaft}

Zum Redaktionsschluss gibt es nur sehr wenige dokumentierte Fälle schwangerer Frauen, die mit COVID-19 infiziert waren. Da die Erkrankung bei einem Großteil der Infizierten mild verläuft, ist auch für die meisten Schwangeren zu vermuten, dass sie nur milde oder moderate Erkältungssymptome entwickeln werden. Es ist jedoch nicht auszuschließen, dass es auch bei schwangeren Frauen zu schweren Krankheitsverläufen kommen kann [15]. Bekannt ist das z. B. für die saisonale und pandemische Influenza, die in der Schwangerschaft mit einem erhöhten Erkrankungs- und Komplikationsrisiko einhergeht. Als Ursache dafür werden verschiedene physiologische und immunologische Veränderungen wie die Verlagerung von zellvermittelter hin zu humoraler Immunität vermutet. Möglicherweise kann diese Veränderung schwangere Frauen empfänglicher für virale Pathogene wie das Influenzavirus machen [1]. Die dokumentierten Fälle schwangerer Frauen mit SARS wiesen auf einen Anstieg der fetalen Sterblichkeit in der Frühschwangerschaft und eine erhöhte maternale Mortalität im weiteren Verlauf der Schwangerschaft hin [13].

Für SARS-CoV-2 ist derzeit ein Fall dokumentiert, bei dem die Mutter nach einer Notsectio und IUFT in der 34. SSW mit Multiorganversagen und ARDS (Acute Respiratory Distress Syndrome) zur Beatmung auf die Intensivstation verlegt werden musste [15][8].
WEB-TIPP

\section{FAQ für Schwangere und ihre Familien}

Das German Board and College of Obstetrics and Gynecology (GBCOG) stellt im Dokument „FAQ für Schwangere und ihre Familien“ Fragen und Antworten zum Thema COVID-19 zusammen. Darunter auch konkrete Verhaltensregeln zum Schutz vor einer Infektion bzw. für vermutete oder bestätigte COVID-19-Infektionen. Das Dokument wird regelmäßig aktualisiert und ist im Internet abrufbar unter: www.dggg.de/fileadmin/documents/Weiter e_Nachrichten/2020/20200312_GBCOG_FAQ_Co rona.pdf

\section{Auswirkungen auf den Fetus}

Bisher sind keine Daten bekannt, die auf eine erhöhte Rate an Fehlgeburten in frühen Phasen der Schwangerschaft durch COVID-19 hindeuten. Da es aktuell keine Hinweise auf intrauterine Infektionen des Fetus mit SARSCoV-2 gibt, sind nach bisherigem Kenntnisstand auch keine kongenitalen Effekte auf den Fetus zu vermuten. Es sind jedoch Fälle von Frühgeburten bei Schwangeren mit COVID-19 beschrieben. Dabei ist bis zum jetzigen Zeitpunkt noch nicht klar, inwieweit diese auf die Viruserkrankung der Frauen zurückzuführen waren [15][8]. 


\section{Auswirkungen auf die Geburt}

Frauen mit vermuteter oder bestätigter COVID-19-Infektion wird geraten, zur Geburt eine Klinik aufzusuchen, in der der Fetus mittels CTG kontinuierlich elektronisch überwacht werden kann. Die Geburt muss in einem isolierten Kreißsaal erfolgen. Der Zustand der Mutter sollte durch Messung der Temperatur, Atemfrequenz und Sauerstoffsättigung überwacht werden [15].

Diskutiert wird derzeit, ob Begleitpersonen für den Kreißsaal weiterhin erlaubt sein sollten. Die DGGG empfiehlt Kliniken, die Begleitung auf den Partner zu beschränken und Besuche auch nach der Geburt nur vom Vater und von Geschwisterkindern zuzulassen. Ob die Empfehlung so beibehalten bleibt, stand zum Redaktionsschluss noch nicht fest und hängt von der Entwicklung der Pandemie in den darauffolgenden Wochen ab [7]. Da es innerhalb der in einem Haushalt lebenden Personen zu einer Übertragung der Infektion kommen kann, sollten begleitende Personen wie der Partner immer einen Mundschutz tragen und sich regelmäßig und gründlich die Hände waschen. Personen mit Symptomen oder bestätigter COVID-19-Infektion sind ausdrücklich aufgefordert, das Haus nicht zu verlassen und dürfen daher nicht mit zur Geburt kommen [15].

Hinweise, dass Frauen mit COVID-19 von einer Periduralanästhesie (PDA), Spinalanästhesie oder Vollnarkose abgeraten werden sollte, gibt es bisher nicht [15]. Zur Verwendung von Lachgas sind verschiedene Angaben zu finden: Die DGGG spricht sich dagegen aus, da Lachgas die Aerosolisierung und damit die Ausbreitung des Virus erhöhen könne [7]. Das RCOG führt in seinem Leitfaden an, dass es keine Beweise für eine Aerosolisierung gäbe. Lachgas solle jedoch immer mit einem mikrobiologischen Filter zur Einmalbenutzung verwendet werden [15].

Aktuell gibt es keinen Anhaltspunkt, dass Frauen mit COVID-19 nicht vaginal gebären sollten. Eine Sectio kann jedoch z.B. bei Atemwegsproblemen durch die Erkrankung indiziert sein. Bis dato existieren keine Hinweise für einen Virusnachweis im Vaginalsekret. Da noch nicht mit Sicherheit gesagt werden kann, ob SARS-CoV2 mit dem Stuhl übertragen werden kann, sollte bei Frauen mit COVID-19 sicherheitshalber auf die Benutzung der Gebärwanne verzichtet werden [15].

\section{WEB-TIPP}

Präventionsmaßnahmen für den Kreißsaal

Mehr Informationen zu geburtshilflichen Präventionsmaßnahmen für Kliniken und Krankenhäuser sind auf der Webseite der DGGG abrufbar unter: www.dggg.de/fileadmin/documents/Weitere_Nach richten/2020/COVID-19_DGGG-Empfehlungen_fuer_ Kreissaele_20200319_f.pdf
Während Literatur aus China die isolierte Unterbringung infizierter Mütter von ihren neugeborenen Kindern vorsieht, empfehlen DGGG und RCOG, die Mutter und das Neugeborene gemeinsam unterzubringen, sofern das Kind gesund ist und nicht neonatologisch überwacht werden muss. Die Vorteile und Risiken sollten dabei mit den Neonatologen und den Eltern individuell abgewogen werden [15][4].

Auch zur möglichen Übertragung von SARS-CoV-2 mit der Muttermilch existieren bisher nur sehr wenige Daten. Die Ergebnisse müssen daher mit Vorsicht bewertet werden. In einer chinesischen Studie waren Proben von sechs getesteten Frauen mit COVID-19 negativ. Das RCOG empfiehlt in seinem Leitfaden daher, dass infizierte Mütter ihre Babys stillen sollten, da davon auszugehen sei, dass die Vorteile des Stillens das Risiko einer potenziellen Übertragung des Virus aufwiegen [15]. Auch das GBCOG empfiehlt infizierten Müttern, ihre Kinder zu stillen. Sie sollten dabei jedoch bestimmte Hygienemaßnahmen einhalten:

- Vor und nach dem Kontakt mit dem Baby, der Milchpumpe oder der Fütterung mit der Flasche ist es wichtig, die Hände zu waschen.

- Während des Stillens ist das Tragen eines Mundschutzes zu empfehlen, um eine Übertragung des Virus durch Tröpfcheninfektion zu vermeiden.

- Bei der Verwendung einer Milchpumpe im Krankenhaus sollte eine spezielle Pumpe verwendet werden.

- Zu Hause sollten nach jedem Gebrauch der Pumpe die Regeln zur Pumpenreinigung strikt befolgt werden.

- Beim Füttern mit Milch oder Milchnahrung wird empfohlen, die Sterilisationsrichtlinien strikt einzuhalten [7].

\section{Empfehlungen für medizinisches Personal}

Damit Ärzte, Hebammen und Krankenpfleger sich bestmöglich vor einer Ansteckung mit SARS-CoV-2 schützen können, empfiehlt das RKI die Verwendung von persönlicher Schutzausrüstung (PSA) bestehend aus Schutzkittel, Einweghandschuhen, dicht anliegender Atemschutzmaske (FFP2 bzw. FFP3 oder Respirator bei ausgeprägter Exposition gegenüber Aerosolen, z. B. bei Bronchoskopie oder anderen Tätigkeiten, bei denen Aerosole entstehen können) und Schutzbrille (14). Die Anforderungen an die persönliche Schutzausrüstung werden in der KRINKO-Empfehlung „Infektionsprävention im Rahmen der Pflege und Behandlung von Patienten mit übertragbaren Krankheiten" spezifiziert [12]. 
WEB-TIPP

Mehr Informationen zum Thema PSA, dem richtigen An- und Ausziehen der Schutzkleidung und Maßnahmen zum Schutz vor kontaktübertragenen Viruserkrankungen sind im Internet zu finden unter: www.thieme.de/de/sonderseiten/corona-schutzaus ruestung.htm

\section{Was jetzt wichtig ist}

\section{Befristete Sonderregelungen für Hebammen}

Neben beruflichen und fachlichen Fragen im Zusammenhang mit COVID-19 treibt viele Hebammen die Sorge um, ob sie durch die fortschreitenden Maßnahmen zur Eindämmung der Corona-Pandemie und die damit verbundenen Einschränkungen die Betreuung der Frauen weiterhin gewährleisten können. Das führt zu Existenznöten. Zudem sorgen sich viele Hebammen um ihre eigene Gesundheit und die ihrer Familie. Der Deutsche Hebammenverband (DHV) hat deshalb für die Zeit der Corona-Pandemie folgende Änderung der Gebührenpositionen erwirkt:

- Die Vorgabe für Dienst-Hebammen max. zwei Leistungen parallel zu erbringen, ist für die Dauer der Vereinbarung ausgesetzt.

- Bestimmte Hebammenleistungen können für die Dauer der Vereinbarung auch digital angeboten werden. Neben der Beratung in Schwangerschaft und Wochenbett sind auch Kurse in digitaler Form abrechenbar.

- Beim Wegegeld wurde die Entfernung von $25 \mathrm{~km}$ auf $50 \mathrm{~km}$ erhöht.

\section{Weitere Informationsangebote}

Der DHV bietet zudem fachliche Unterstützung für alle Fragen rund um den Umgang mit dem Coronavirus, Hinweise zur Hebammenausbildung und zum Studium und kündigt einen Kurs mit aktuellem Hebammenwissen zur Betreuung während der Corona-Pandemie in der Lernplattform „Olga“ an (www.hebammenverband-olga.de).

\section{Versorgung mit Schutzkleidung}

Am 21.03.2020 kündigte der DHV auf seiner Webseite an, dass bald die dringend benötigte Schutzkleidung für Kreißsäle, Geburtshäuser und Hebammenpraxen verfügbar sein werde. Um die flächendeckende Versorgung sicherzustellen, werde der DHV in Abstimmung mit seinen Landesverbänden die notwendigen Bedarfe für die nächsten Monate an das zuständige Beschaffungsamt des Bundesministeriums des Innern weitergeben und die Schutzkleidung dann entsprechend verteilen. 


\section{WEB-TIPP}

\section{Hebammenverband informiert}

Alle aktuellen Informationen des Deutschen Hebammenverbandes zum Thema sind im Internet abrufbar unter www.hebammenverband.de

\section{Zum Weiterlesen}

Eine Übersichtsarbeit ist in der Zeitschrift Geburtshilfe und Frauenheilkunde erschienen: Stumpfe FM, Titzmann A, Schneider MO et al. SARS-CoV-2-Infektion in der Schwangerschaft; Geburtsh Frauenheilk 2020, doi: https://doi.org/10.1055/a-1134-5951

\section{Autorinnen / Autoren}

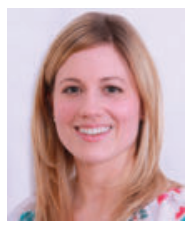

Marion Anna Becker ist freie Journalistin und hat sich auf Gesundheitsthemen spezialisiert.

Korrespondenzadresse

Marion Anna Becker
Höhestraße 34
61348 Bad Homburg
E-Mail: m.becker@gesund-informiert.de

\section{Literatur}

[1] Charité Universitätsmedizin Berlin. Influenza (21.03.2020). Im Internet: https://www.embryotox.de/erkrankungen/details/ influenza

[2] Chen $\mathrm{H}$, Guo J, Wang $\mathrm{C}$ et al. Clinical characteristics and intrauterine vertical transmission potential of COVID-19 infection in nine pregnant women: a retrospective review of medical records. Lancet 2020, doi: https://doi.org/10.1016/ S0140-6736(20)30360-3

[3] Chen S, Huang B, Luo DJ et al. Pregnant women with new coronavirus infection: a clinical characteristics and placental pathological analysis of three cases. Zhonghua Bing Li Xue Za Zhi 2020; 49(0): E005-E05, doi: 10.3760/cma.j.cn11215120200225-00138

[4] Deutsche Gesellschaft für Gynäkologie und Geburtshilfe (DGGG). Empfohlene Präventionsmaßnahmen für die geburtshilfliche Versorgung in deutschen Krankenhäusern und Kliniken im Zusammenhang mit dem Coronavirus (20.03.2020). Im Internet: https://www.dggg.de/fileadmin/documents/Wei tere_Nachrichten/2020/COVID-19_DGGG-Empfehlungen_fuer Kreissaele_20200319_f.pdf; Stand: 19.03.2020

[5] Deutsches Zentrum für Infektionsforschung (DFIZ): Coronavirus (20.03.2020). Im Internet: https://www.dzif.de/de/glos sar/coronavirus
[6] European Centre for Disease Prevention and Control. COVID19 (20.03.2020). Im Internet: https://www.ecdc.europa.eu/e n/novel-coronavirus-china; Stand: 20.03.2020

[7] German Board and College of Obstetrics and Gynecology. FAQ für schwangere Frauen und ihre Familien (20.03.2020). Im Internet: https://www.dggg.de/fileadmin/documents/Wei tere_Nachrichten/2020/20200320_GBCOG_FAQ_Corona.pdf; Stand: 20.03 .2020

[8] Liu $\mathrm{Y}$, Chen $\mathrm{H}$,Tang $\mathrm{K}$ et al. Clinical manifestations and outcome of SARS-CoV-2 infection during pregnancy. Journal of Infection 2020, doi: https://doi.org/10.1016/j. jinf.2020.02.028

[9] Neuartiges Coronavirus (SARS-CoV-2). Deutsche Medizinische Wochenschrift 2020; 145(5):336-337, doi:10.1055/a-11133096

[10] Robert Koch-Institut: COVID-19: Jetzt handeln, vorausschauend planen. Strategie-Ergänzung zu empfohlenen Infektionsschutzmaßnahmen und Zielen 2. Update. Epid Bull 2020; 12: 3-6, doi: $0.25646 / 6540.2$

[11] Robert-Koch-Institut (RKI). Empfehlungen des Robert KochInstitutes zu Hygienemaßnahmen im Rahmen der Behandlung von Patienten mit einer Infektion durch SARS-CoV-2 (20.03.2020). Im Internet: https://www.rki.de/DE/Content/In fAZ/N/Neuartiges_Coronavirus/Hygiene.html; Stand: 19.03.2020

[12] Robert-Koch-Institut (RKI). Infektionsprävention im Rahmen der Pflege und Behandlung von Patienten mit übertragbaren Krankheiten. Empfehlung der Kommission für Krankenhaushygiene und Infektionsprävention (KRINKO) beim Robert Koch-Institut (20.03.2020). Im Internet: https://www.rki.de/ DE/Content/Infekt/Krankenhaushygiene/Kommission/Downlo ads/Infektionspraev_Pflege_Diagnostik_Therapie.pdf?_blo b=publicationFile; Stand: 28.09.2015

[13] Robert-Koch-Institut (RKI). Krankheitsbeschreibung von SARS (21.03.2020). Im Internet: https://www.rki.de/DE/Content/In fAZ/S/SARS/Klinik.html; Stand: 23.10.2003

[14] Robert-Koch-Institut (RKI). SARS-CoV-2 - Steckbrief zur Coronavirus-Krankheit-2019 (COVID-19) (20.03.2020). Im Internet: https://www.rki.de/DE/Content/InfAZ/N/ Neuartiges_Coronavirus/Steckbrief.htm; Stand: 13.03.2020

[15] Royal College of Obstetricians and Gynaecologists (RCOG). Coronavirus (COVID-19) Infection in Pregnancy. Information for healthcare professionals. Version 4. (21.03.2020). Im Internet: https://www.rcog.org.uk/globalassets/documents/ guidelines/2020-03-21-covid19-pregnancy-guidance-2118.p df: Stand: 18.03.2020

[16] World Health Organization (WHO). Novel Coronavirus (SARSCoV-2) situation reports (20.03.2020). Im Internet: https://w ww.who.int/emergencies/diseases/novel-coronavirus-2019/si tuation-reports/; Stand: 20.03.2020

[17] World Health Organization (WHO). Report of the WHO-China Joint Mission on Coronavirus Disease 2019 (COVID-19) (20.03.2020). Im Internet: https://www.who.int/docs/defaul t-source/coronaviruse/who-china-joint-mission-on-covid-19-fi nal-report.pdf; Stand: 24.02.2020

\section{Bibliografie}

DOI https://doi.org/10.1055/a-1110-2007

Die Hebamme 2020; 33: 17-22

(c) Georg Thieme Verlag KG Stuttgart · New York ISSN 0932-8122 